\title{
EL AUGE HISTÓRICO DE LA ESTILÍSTICA Y SU IMPRONTA EN COSTA RICA
}

\author{
Carlos Manuel Villalobos
}

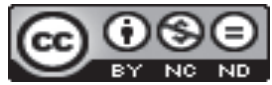

Doi: https://doi.org/10.15517/rfl.v46iExt..43484

URL: https://revistas.ucr.ac.cr/index.php/filyling/index 



\title{
EL AUGE HISTÓRICO DE LA ESTILÍSTICA Y SU IMPRONTA EN COSTA RICA
}

\author{
THE HISTORICAL BOOM OF STYLISTICS \\ AND ITS IMPRINT IN COSTA RICA
}

Carlos Manuel Villalobos

\begin{abstract}
RESUMEN
En este artículo, se estudia el impacto histórico de la estilística y los efectos que tuvo en Costa Rica. Se parte de una publicación hecha por Manuel Picado en 1975 donde resume los postulados de la estilística según Amado Alonso. Esta metodología de análisis de la expresión verbal surgió a inicios del siglo XX como parte de la lingüística estructural, pero sus mayores aplicaciones fueron en el ámbito de los estudios literarios. A través del estilo, manifiesto en la forma, se accede al espíritu creador, ya sea para valorar a un autor específico o para caracterizar un grupo cultural. En el artículo, se estudia su paso por las escuelas idealistas de Alemania y España y se hace referencia al auge que tuvo en Hispanoamérica. En la última parte, se analiza la huella que dejó en los estudios literarios costarricenses: desde el estudio del estilo en el marco de la prescriptiva literaria hasta los primeros atisbos estilísticos con Roberto Brenes Mesén y Arturo Agüero Chaves. En este contexto, destaca el trabajo de Abelardo Bonilla de 1967, quien intenta explicar, con ejemplos literarios, el estilo del espíritu costarricense.

Palabras clave: estilística; historia de la estilística; estilística costarricense; teoría literaria; Amado Alonso.
\end{abstract}

\begin{abstract}
This paper studies the historical impact of stylistics and its effects in Costa Rica. It is based on a publication made by Manuel Picado in 1975 where he summarizes the postulates of stylistics according to Amado Alonso. This methodology of analysis of verbal expression emerged in the early twentieth century as part of structural linguistics, but its main applications were in the field of literary studies. Through the style, manifested in the form, the creative spirit is accessed, either to value a specific author or to characterize a cultural group. The paper studies its passage through the idealist schools of Germany and Spain and the boom it had in Latin America. In the last part, is analyzed the mark that it left in Costa Rican literary studies: from the study of style in the framework of the literary prescriptive to the first stylistic glimpses with Roberto Brenes Mesén and Arturo Agüero Chaves. In this context, the work of Abelardo Bonilla of 1967 stands out. He tried to explain, with literary examples, the style of the Costa Rican spirit.

Keywords: stylistics; history of stylistics; Costa Rican stylistics; literary theory; Amado Alonso.
\end{abstract}

Dr. Carlos Manuel Villalobos. Profesor de la Escuela de Filología, Lingüística y Literatura. Universidad de Costa Rica. San José, Costa Rica.

Correo electrónico: carlos.villalobos@ucr.ac.cr 


\section{Delimitaciones de la estilística}

En 1975, en el segundo número de la Revista de Filología y Lingüística, Manuel Picado publica un artículo en el que resume los postulados básicos de la estilística según la propuesta del pensador hispano argentino Amado Alonso (1896-1952). Para ese momento, según Picado, los estudios estilísticos habían sido objeto de diversos cuestionamientos; no obstante, considera que los aportes de Alonso son valiosos y esto justifica una formulación adecuada de sus propuestas (1975, p. 97). Este artículo forma parte del trazo histórico que dejó la estilística en el campo de los estudios lingüísticos y literarios en Costa Rica. Parto de la premisa de que no se trata de un trabajo aislado en relación con dicha disciplina. En la primera mitad del siglo XX, el estudio del estilo formaba parte de la preceptiva literaria que se enseñaba en secundaria y la estilística, como campo gnoseológico, fue considerada en varios textos académicos.

En el marco de la conmemoración de ese segundo número de la Revista de Filología y Lingüística y del artículo en cuestión, me propongo rastrear la huella de la estilística en Costa Rica: ¿Quiénes y de qué manera la consideraron? ¿Qué textos dieron cuenta de la disciplina y cuáles fueron las corrientes que se utilizaron? ¿Qué implicaciones tuvo la estilística en el campo de los estudios filológicos, linguiísticos y literarios? Para comprender el contexto epistemológico e histórico de la estilística, de entrada, son necesarias algunas precisiones generales que expongo a continuación.

Aunque el estudio del estilo es un ejercicio analítico del discurso que data de la antigüedad y forma parte del quehacer de la retórica grecolatina y, en particular, de la preceptiva literaria ${ }^{1}$, la estilística tal y como se conoce a inicios del siglo XX nace en el ámbito de la linguíística. Se atribuye su constitución a las lecciones de Ferdinand de Saussure (1857-1913), el fundador de la lingüística estructural, quien establece la diferencia entre habla y lengua. La lengua implica un sistema social que se proyecta sobre lo personal, es decir, sobre el habla. Esta distinción es la que le permite a su discípulo Charles Bally (1865-1947), proponer que es posible el estudio del estilo de cada lengua en el marco de una delimitación histórica. Su primer acercamiento es un estudio que titula Tratado de estilística francesa, publicado en 1909; pero es en el libro El lenguaje y la vida de 1913, donde define con más precisión el campo de la nueva disciplina. Para Bally, la estilística debe abarcar todos los fenómenos

1 La preceptiva literaria es una formalización académica que estudia la literatura a partir de preceptos derivados de la retórica y la poética clásicas. Incluye el estudio del estilo, entendido como el modo particular en que cada escritor expresa su pensamiento. Considera que el estilo estético, trabajado bajo los principios de las reglas retóricas, garantiza la calidad literaria. Por esta razón, la preceptiva defiende un estilo de corrección ajustado a la norma. Estas pautas se enseñaron en los sistemas educativos de tradición europea más o menos hasta mediados del siglo XX. Se imprimían manuales que en general repetían los mismos esquemas, aunque cada autor solía ajustar los ejemplos a las literaturas locales. En todos los países de Centroamérica, por ejemplo, desde 1885 se utilizó en varios sistemas escolares un texto elaborado por el salvadoreño Francisco Castañeda, titulado Lecciones de Retórica o Literatura Preceptiva (1948). Siguiendo el mismo modelo, uno de los últimos manuales del género es el que publica el guatemalteco Enrique Muñoz Meany en 1951. En el capítulo referente al tema del estilo, quizá por influencia de la estilística -aunque no lo aclara- Muñoz es consciente de otras acepciones que no solían ser incluidas en los manuales anteriores. En este caso, además, de las acepciones referentes a lo retórico, alude al estilo histórico. Señala que: "Así como el hombre posee una fisonomía que lo caracteriza, también cada época, cada raza y cada país, tienen una individualidad propia, la cual influye poderosamente en los estilos de los escritores, dándoles una modalidad o un carácter común” (1951, p. 154). 
lingüísticos, pues cada hecho del lenguaje "puede manifestar alguna parcela de la vida del espíritu y algún movimiento de sensibilidad” (1962, p. 102). Propone que este estudio integral incluya los siguientes niveles lingüísticos: fonología, vocabulario y sintaxis. Condiciona el estudio a una sincronía específica; es decir, a un período determinado, "sin permitirse buscar materiales o pruebas en los periodos anteriores o subsiguientes; (y) debe aplicarse a todas las manifestaciones de la vida lingüística del idioma" (1962, p. 121). El vínculo entre el estilo de la lengua y lo emocional, hacen que la propuesta de Bally se fundamente en principios idealistas que no estaban presentes en la Filología del siglo XIX. Por eso, según acota José Luis Martín, "hoy día, esta disciplina concibe el lenguaje como una expresión del espíritu, que moldea la lengua a sus necesidades" (1973, pp. 147-148).

Si bien, se considera a Bally como el fundador moderno de la estilística, a esta tarea hay que agregar los aportes de Benedetto Croce (1866-1952), quien refuerza la perspectiva idealista, centrada en la tesis de que el espíritu humano se manifiesta en la historia del lenguaje y sus manifestaciones artísticas, lógicas, económicas o éticas. Estas ideas son retomadas por dos teóricos alemanes que ahondan en la concepción intuitiva de la creación literaria. Son Karl Vossler (1872-1949) quien, a diferencia de Bally, defiende la consideración de los elementos históricos del lenguaje y Leo Spitzer (1887-1960), quien desarrolla la estilística genética del individuo y, por lo tanto, se desliga de la noción totalizadora de los lingüistas francófonos. Spitzer propone un método que llama "círculo filológico", que parte de los postulados de la crítica inmanente y la idea de que el autor es el centro o el motor que mueve la obra. Sostiene que lo central en un texto literario son los detalles lingüístico-estilísticos y que estos se relacionan directamente con el autor. Propone que para acceder a la obra es necesario un salto intuitivo que capte el procedimiento estético. Para lograrlo es necesario considerar las generalidades periféricas y relacionarlas con las especificidades centrales. Además, la crítica "debe hacerse en sentido de simpatía y empatía con la obra misma y con su autor" (Martín, 1973, p. 155). En resumen, el método spitzeriano considera dos fases: la inductiva, que propone una hipótesis posible mediante un salto intuitivo y la deductiva, donde se verifica la hipótesis inicial.

Hasta aquí, el campo de la estilística, a pesar de sus coincidencias idealistas, se divide en dos corrientes básicas: la descriptiva o estilística de la expresión que se fundamenta en el análisis de la lengua según los postulados de Charles Bally, y la genética o estilística del individuo que, al centrarse en el fenómeno del autor y la obra literaria, prioriza el habla, tal y como lo propone Leo Spitzer. Posteriormente, aparecerán otras teorías estilísticas, tales como la funcionalista de Michael Rifaterre (1924-2006) quien ahonda en la intencionalidad del autor y agrega a la metodología el concepto de archilector, el cual consiste en la revisión de lecturas previas que hayan valorado el estilo (Gómez, 1999. p. 90). Otra corriente es la estructuralista de Jean Cohen, quien para el análisis estilístico, considera los niveles de la expresión y el contenido o los planos fónico y semánticos (Gómez, 1999, p. 97).

\section{La escuela española}

En España, Ramón Menéndez Pidal (1869-1968) inicia el interés por el estilo desde una perspectiva lingüística y filológica, pero serán Amado Alonso (ya citado) y Dámaso Alonso (1898-1990) quienes desarrollen los estudios estilísticos con mayor profundidad, en concordancia con el modelo esteticista literario de la corriente alemana. Ambos estudiosos 
discuten la dicotomía fondo y forma, la búsqueda de la belleza en la creación poética, así como el estudio del sistema expresivo de la obra y el análisis de su estructura interna y externa (Martín, 1973, p. 160).

Aprovecho aquí la sistematización que hace Manuel Picado en su artículo de 1975 para ofrecer otras precisiones a propósito del pensamiento de Amado Alonso y su concepción de la estilística genética. Al respecto, el profesor costarricense divide su esbozo en siete apartados. El primero está dedicado al ideario estético. Retoma los postulados idealistas de la estilística que sigue Amado Alonso, quien entiende la literatura como goce estético. Por esta razón, frente a la dicotomía entre fondo y forma, lo fundamental será la estructura, pues es en la composición de la obra donde se concreta la belleza. De seguido incluye el tema de lo poético, pues la estilística se avoca casi de manera exclusiva a la lírica. Lo poético se entiende como un proceso dialéctico en el que confluyen los sentimientos y la intuición. En esta delimitación idealista, calza la tesis platónica de la inspiración, por ello, la poesía se desliga del conocimiento racional y se resiste a ser interpretada por un conocimiento meramente científico.

Con base en una carta que Amado Alonso le remite al mexicano Alfonso Reyes, Manuel Picado explica, en un tercer punto, la diferencia entre la estilística de la lengua y la del habla. La primera, se refiere a los contenidos de las formas comunales y la segunda, a las expresiones que se concretan en el habla. Se trata de los indicios que dan cuenta de lo afectivo, lo activo, lo fantástico y lo valorativo. Desde luego que ambas perspectivas tienen entre sí una relación de precedencia. El apartado número cuatro está dedicado a la delimitación y al objeto de la estilística literaria. Lo esencial en la tarea interpretativa es hallar los rasgos del estilo que subyace en la "intimidad" de la obra; en otras palabras, encontrar las claves síquicas de la expresión. El axioma de base consiste en la tesis de que hay una armonía entre la expresión verbal y el conjunto de la obra. Como consecuencia, el objeto de estudio estilístico consiste en mostrar el procedimiento de las fuerzas síquicas que componen la obra y alcanzar el goce estético mediante la contemplación de la forma poética.

En un quinto punto, Picado plantea la diferencia entre la estilística y la crítica tradicional. Este último corresponde a la perspectiva filológica enunciada a principios del siglo XIX por Friedrich A. Wolf. De acuerdo con el pensador alemán, para la interpretación de una obra literaria es necesario abordar tópicos tales como las costumbres de la época, las ideas, la mitología, la geografía referida, los sistemas filosóficos, las particularidades gramaticales, la vida social y política, así como las condiciones personales del autor. En contraposición, Amado Alonso cree que este enfoque omite la especificidad estética de la obra. La estilística no rechaza los aportes de la filología, pero los utiliza si estos permiten acceder a la belleza de la forma. De este modo, "si la crítica tradicional ha puesto en primer plano cuáles son las fuerzas históricas que se juntan y armonizan en el autor estudiado, la estilística estudia esa concurrencia en cuanto material de creación" (Picado, 1975, p. 100).

El siguiente apartado, que se titula "Actitud del estudioso del estilo", explora dos preguntas fundamentales que plantea Alonso relativas al procedimiento interpretativo: “¿Cómo está constituida la obra? y ¿qué delicia estética provoca?”. El primer interrogante se refiere al producto creado, y el segundo, a la actividad creadora. De ahí que la estilística, además de la forma, aborde el modo de sentir o la fuerza síquica del autor. Por esto, es necesario vincular la información biográfica con el sentido de la obra. En el último punto, Picado se refiere al desarrollo del método y, para ello, pone como ejemplo el libro Poesía y estilo de Pablo Neruda, 
donde Amado Alonso desarrolla, de manera más amplia, la metodología estilística. En la parte inicial, con la intención de captar la totalidad, hace un examen de los procedimientos expresivos. Luego, aborda la dialéctica sentimental-intuitiva para mostrar el ensimismamiento nerudiano y la experiencia de la realidad en constante destrucción. Agrega a este análisis el estudio del ritmo, la sintaxis y la presencia de la forma.

\section{El impacto de la estilística en América Latina}

La estadía de Amado Alonso ${ }^{2}$ en Buenos Aires y su prestigio académico, similar al de Dámaso Alonso, incide en el interés que suscita la estilística en Latinoamérica ${ }^{3}$, aunque no todos los que abordan la disciplina asumirán ad portas los postulados idealistas. En Argentina, entre otros autores destacan Ángel J. Battistessa (1902-1993), discípulo de Dámaso. En 1965 publica El poeta y su poema, con un prólogo de su maestro. También Enrique Anderson Imbert (1910-2000) muestra interés por la disciplina en varios de sus trabajos sobre teorías literarias. Sin embargo, la mayor contribución la hará Raúl Castagnino (1914-1999), en varios de sus libros; sobre todo, con un texto titulado El Análisis literario: introducción metodológica a una estilística integral, cuya primera edición es de 1953. Este libro de Castagnino resulta novedoso en el contexto de las aportaciones críticas de la disciplina pues intenta ir más allá de las cuestiones estéticas y sugiere que, para un análisis integral, la estilística debe ocuparse también del lenguaje coloquial, lo psicológico y todo aquello que permita iluminar el sentido. De ahí que para comprender una obra literaria es necesario captar el mundo que subyace y descubrir el modo en que cada escritor le imprime a las palabras un nuevo color. Las palabras no solo tienen un sentido que capta el intelecto, "sino que también sugieren color, sabor, olor, matiz, movimiento, temperatura, estados, etc.; todo lo puede producir o insinuar el creador literario y todo lo debe reproducir quien pretende comprender una obra" (1971, p. 25).

En México, Alfonso Reyes (1889-1959), en algunas de sus obras, también reflexiona sobre los alcances de la estilística, pero, por cierto, de una manera bastante crítica. Por ejemplo, en El deslinde: prolegómenos a la teoría literaria (1944) propone que el estilo, en su vinculación con lo idiomático, se enfrenta a dos problemas: en lo interno, debe ajustarse a las

2 De acuerdo con Fernández Retamar: "El magisterio de Amado Alonso se tradujo en un discipulado con frecuencia admirable que ha renovado la crítica americana. Bástenos mencionar a Raimundo Lida, a quien debemos estudios excelentes sobre Quevedo- uno de los primeros trabajos estilísticos en nuestra lengua-, Juan Ramón Jiménez Darío, a María Rosa Lida, cuyo libro sobre Juan de Mena ha sido considerado por Hatzfeld una obra esencial en la evolución de la moderna estilística; a Enrique Anderson Imbert y a Juan Carlos Ghiano, que han realizado, entre otros, estudios estilísticos de Montalvo y Lugones, respectivamente" (1958, p. 121).

3 José Luis Martín (1973) ofrece una lista de autores latinoamericanos que han abordado el tema de la estilística. Entre estos están: Raimundo Lida, María Rosa Lida, Eleuterio F. Tiscornia, Ángel Resenblat, Hugo Rodríguez Alcalá, Anfredo A. Roggiano, Luis Monguió, Eugenio Florit, José Antonio Portuondo, Gabriela Mistral, Carmelo M. Bonet, Rodolfo Oroz, Pedro Henríquez Ureña, José A. Balseiro, Margot Arce, Cesáreo Roa Nieves, Luis Leal, Marcos Morínigo, Ana María Barrenechea, Pereira Rodríguez, Emilio Carilla, Cintio Vitier, Borges, Silvio Zavala, Cecilia Hernández, Ernesto Mejía Sánchez, Antonio Alatorre, Martín Luis Guzmán y Andrés Uduarte. Curiosamente en esta lista no aparece Fernández Retamar. Se trata, en todo caso, de una lista parcial, pues no aparecen acá los centroamericanos Matilde Elena López, Elsie Alvarado o Abelardo Bonilla, entre otros autores que también se refirieron a la estilística en sus investigaciones académicas. 
determinaciones psicológicas del literato y, en lo externo, a las cuestiones estéticas. Por esta razón, "al estilo se llega por entre una maraña de desajustes" (1944, p. 227). Desconfía de la capacidad del lenguaje para dar cuenta de la carga subjetiva y testificar sobre la intención del autor. No obstante, considera que

[...] a la eficacia del estilo conviene esta vida peligrosa de los desajustes, este sentirse siempre a un paso del abismo. El ajuste ha de ser logro momentáneo y nunca de antemano seguro. Un ajuste institucional y garantizado sería la ruina de la literatura, ahogaría los posibles escapes de la fuerza expresiva (1944, p. 228).

En 1945, en su libro Tres puntos de exegética literaria, Reyes propone que para la creación de la Ciencia de la literatura es necesario integrar el método histórico, el psicológico y el estilístico, de este modo, a pesar de los cuestionamientos, acredita el análisis del estilo como una operación válida para el estudio de la literatura.

En Cuba, en 1958 Roberto Fernández Retamar publica el libro Idea de la estilística. Se trata de una sistematización que inicia con la discusión sobre los vínculos históricos de la estilística con la Retórica y la Filología. Explora sus orígenes actuales en el marco de la lingüística estructural y establece la clásica diferenciación entre lengua y habla para explicar las dos actitudes de los estudios estilísticos. Retamar asume una postura teórica que coincide con el enfoque estético de los idealistas alemanes y españoles. Considera que es un contrasentido limitar el estilo a la lengua tal y como lo propone Charles Bally, y se identifica con la enmienda de Vossler, pues es en el ámbito del individuo donde es posible identificar el estilo (1958, p. 76). Asegura que su intención es presentar "el mejor rostro" de la estilística, pero desea formular algunas objeciones. No está de acuerdo con las aplicaciones mecánicas de algunas metodologías y tampoco con la tendencia a excluir la circunstancia histórica de las obras literarias, "dedicándose a estudiarlas al margen del tiempo, lo que las priva de sentido" (1958, p. 135). Finalmente, critica lo que denomina como "la ósmosis crítica" en referencia a la actitud de algunos estudiosos que al pretender dar cuenta del estilo literario caen en el impresionismo (1958, p. 138).

En el ámbito centroamericano, aparte del caso costarricense que analizaré en el siguiente apartado, hay que considerar los aportes de al menos dos profesoras que publicaron trabajos académicos que incluyen la estilística como marco teórico metodológico. Se trata de la salvadoreña Matilde Elena López (1919-2010) con libros como Interpretación social del arte (1974) y Estudios sobre poesía (1973), y la panameña Elsie Alvarado de Ricord (1928-2005), quien entre otras obras publicó: Estilo y densidad en la poesía de Ricardo J. Bermúdez (1960), La obra poética de Dámaso Alonso (1968), Aproximación a la poesía de Ricardo Miró (1973) y Rubén Darío y su obra poética (1978).

Para Elsie Alvarado de Ricord, en la tarea interpretativa del texto literario los factores subjetivos son tan significativos como los ambientales. Por esto, el estudioso con una actitud crítica consciente puede tomar una posición "configurada a la luz o a la sombra de una convicción política, y es posible que luche por trasplantar al terreno artístico los criterios que son válidos en otros campos de la cultura" (1973, p. 25). Esta actitud se enmarca en lo que la autora define como la enumeración caótica necesaria para activar las diversas implicaciones de la obra. De este modo, las posturas idealistas de la estilística se mantienen vigentes en la propuesta de la profesora panameña. Acepta que el significado artístico tiene un carácter metafórico, sumamente polisémico y esto le confiere a la literatura un dinamismo esencial. "Ese fluir anímico inasible es a la poesía lo que el color es a las artes figurativas y la melodía 
a la música” (1973, p. 27). Por ello, la percepción emocional del lector con algún nivel de sensibilidad es clave para asegurar el éxito hermenéutico. De acuerdo con Alvarado, para acceder a este "fluir anímico" el mejor camino es el que propone la estilística. Defiende el estudio del estilo en los siguientes términos:

La razón del estilo es la razón interna de toda la poesía, de la personalidad del autor. Cuanto más se detalle y analice el estilo más se penetrará en el sentido de los temas, en la evolución intelectual, emotiva y estética del bardo, y más se conocerá su concepción del mundo y de los móviles profundos que condicionan su actitud ante la vida, inclusive su conducta social $(1960$, p. 28).

La tesis de la sensibilidad crítica del lector encuentra menos eco en la obra de Matilde Elena López. La salvadoreña considera la crítica estilística, pero con una fuerte orientación sociológica. Según su propuesta:

\footnotetext{
Es entendido que una línea estilística en la que se ordenan las formas de expresión individuales, a primera vista parece libremente escogida, pero ella reviste frente a los esfuerzos individuales mucho más intensamente que las tendencias histórico-sociales, el carácter de una LEY OBJETIVA, de una LEY SOCIAL. Retrospectivamente los diversos individuos se nos aparecen como soportes de tendencias estilísticas anónimas (1974, p. 14).
}

Para desarrollar un análisis crítico, López acepta que se puede partir del método estilístico y para ello, el crítico debe hallar primero el núcleo de partida, que puede ser una pista imprecisa o una intuición, pero necesariamente es requisito que, acto seguido, se someta a comprobaciones rigurosas. "Esa intuición es para el crítico, lo que es el don del diagnóstico para el médico" (1974, p. 29). Con base en las ideas de Dámaso Alonso, López propone tres tipos de conocimiento poético: el del lector, el crítico y el científico. El primero recibe el impacto poético; el segundo tiene la capacidad de discernir la autenticidad de la obra, y el tercero es el que corresponde a la nueva tarea de la estilística (Villalobos, 2010, p. 55). En palabras de López:

La búsqueda del tercer conocimiento poético (en esencia distinto de los anteriores) se realiza mediante métodos estilísticos. Pero estos tres conocimientos son como tres escalones. Nadie podrá ser investigador de la Estilística que no haya sido primero un apasionado lector y un intenso crítico (1974, p. 510).

A diferencia de Alvarado, López exige un lector mucho más preparado culturalmente, pues el estilo no solo incluye una dimensión personal, sino también lo genérico y la época. El estilo individual se refiere a las particularidades personales del autor; el de género, a las condiciones de enunciación genérica, y el de época constituye un rasgo histórico estructural que se da de antemano en todas las obras de un momento histórico (1974, p. 14). La prioridad histórico contextual que propone López muestra su inclinación por la llamada estilística externa, mientras que Alvarado se decanta por la genética individual o interna.

Ambas estudiosas consideran, sin embargo, que el crítico no debe atenerse solo a un método. Aceptan la flexibilidad metodológica y pasan de la estilística al estructuralismo. Matilde Elena López plantea que "es indiscutible que la obra de arte posee su propia lógica inmanente y que su peculiaridad se exprese de la manera más clara en las relaciones estructurales internas, entre los distintos estratos y elementos formales de la obra artística" (1974, p. 31). Elsie Alvarado de Ricord, por su parte, plantea que "La obra literaria en sí es, ciertamente, un sistema relativamente autónomo, de signos. Estos tienen doble cara: significado y significante; de la relación entre ambos resulta su funcionalidad" (1973, p. 25). Estas acotaciones evidencian que, para el contexto de producción de sus trabajos, la estilística empezaba a perder terreno frente a otras tecnologías de análisis literario. 


\section{La estilística en Costa Rica}

En su artículo de 1975, Manuel Picado advierte que las ideas del método que sigue Amado Alonso resultan discutibles para esa fecha y aclara que sus acotaciones no deben interpretarse como un gesto de adhesión incondicional, "sino más bien como un paso necesario para cualquier trance crítico ulterior” (1975, p. 97). Reconoce que el proyecto teórico de Alonso tiene logros que deben ser respetados, sobre todo en el ámbito de la lírica.

De acuerdo con Cristina D'Alton (1990) la estilística sirvió de puente entre los modelos descriptivos e interpretativos, pues consideró el análisis de las estructuras rítmicas y sonoras, el vocabulario y los giros verbales, la sintaxis y, en general, las particularidades lingüísticas; pero no se detuvo ahí ya que intentó vincular estos rasgos con la expresión de una realidad histórica ligada a un estado psicológico (1990, p. 86). Este procedimiento abarcador garantizó su éxito durante varias décadas, pero, a mediados del siglo XX, empezó a ser sustituido por modos de análisis más rigurosos. Por esto, en Costa Rica para 1975, al igual que en otros contextos, la estilística ya era parte de la historia del abanico de opciones que había tenido la teoría literaria en ese siglo. La revisión que hace Picado en el citado artículo no es única: varios estudiosos costarricenses previamente habían aludido a la metodología en sus escritos. También habrá otras referencias posteriores.

Para situar la impronta del estudio del estilo en Costa Rica y más adelante de la estilística es necesario remontarse a los antiguos manuales de retórica y preceptiva literaria. Esta herencia será retomada por los profesores de secundaria encargados de redactar los textos oficiales de la enseñanza pública. Destaca en esta tarea el trabajo del profesor Hernán Zamora Elizondo (1895-1967), quien en 1929 publica el libro Orientación Literaria. De acuerdo con la acotación liminar que aparece al inicio, el texto se ajusta al programa de preceptiva literaria para la educación secundaria por acuerdo oficial del 28 de octubre de ese año. Como corresponde en este tipo de manuales, la enseñanza del estilo es tema obligatorio. Aunque Zamora lo define como el modo personal de cada artista, le atribuye el requisito estético de la tradición preceptiva; es decir, su vinculación con el conocimiento de la técnica retórica. De este modo solo los grandes artistas consiguen un estilo propio. De acuerdo con Zamora:

\footnotetext{
Los aprendices prueban todas las modalidades, ensayan todos los métodos, imitan todos los modelos, esto es natural y aun conveniente, es el afán de la burbuja de vapor que busca salida hacia la amplitud del espacio: pero el artista ya hecho se caracteriza siempre por un estilo suyo que lo individualiza (1931, p. 23).
}

Esta misma concepción tradicional es la que defiende Moisés Vincenzi (1895-1964) en dos obras que también tienen pretensiones didácticas. Se trata de La enseñanza del estilo de 1939 y Preceptiva Literaria y Composición de 1940. En el primer libro ensaya recursos normativos para la enseñanza de la redacción y en el segundo reproduce las categorías habituales de la preceptiva literaria. Sobre el tópico del estilo argumenta que solo el genio es capaz de modelar una escritura original. Al respecto apunta:

El estilo será siempre una de las más arduas preocupaciones de los escritores de sangre ilustre, de los verdaderos estetas, quienes trabajarán en una labor benedictina por darle la suavidad de la seda, la limpieza del jaspe, el centelleo de las gemas, la instrumentación verbal (1940, pp. 122-123).

En los planteamientos de los profesores Zamora y Vincenzi se evidencia una visión idealista que reproduce, en su concepción del estilo, el enfoque individual de la estilística, pero 
hasta esta fecha las acotaciones reproducen esquemas más cercanos al impresionismo ${ }^{4}$ y no se preocupan por el análisis propiamente de la obra o el estudio específico de un autor.

El texto que rompe de manera consciente con estas concepciones tradicionales se titula Estilística del lenguaje costarricense (1967) de Abelardo Bonilla Baldares (1898-1969). En la introducción, Bonilla acota que conoce dos trabajos previos al suyo que han sido elaborados desde los esquemas de la estilística. Se trata del artículo "El ritmo de la prosa española" de Roberto Brenes Mesén (1874-1947), publicado en 1938 en la Revista Hispania y el "Estudio epilogal" del libro Cifra antología de Fabio Baudrit González (1956) de Arturo Agüero Chaves (1907-2001).

El trabajo de Roberto Brenes Mesén no es un análisis estilístico que dé cuenta de un autor particular, se trata de un ambicioso proyecto en el que busca determinar el ritmo general de la prosa española. Mediante una metodología de vocalización analizó la regularidad de los períodos en un conjunto de obras y contabilizó el estilo de más de treinta escritores. Empezó el proyecto en 1919 y, con ayuda de un grupo de estudiantes, lo concluyó en 1937. Encontró que la media del período de la prosa concuerda con "los mismos periodos rítmicos que hallamos en los viejos romances y en el diálogo de damas y galanes y graciosos de los siglodorados (sic) dramaturgos de nuestra lengua" (Brenes Mesén, 1974, p. 400). Es curioso que este proyecto prosódico estuviera entre los planes académicos de Brenes Mesén, pues parece contradecir las aseveraciones que aparecen en su libro Las categorías literarias de 1923, donde se opone a los análisis retóricos y a las metodologías dedicados a la descripción. Afirma que, así como al gramático, "al retórico se le escapa la concepción del conjunto, la emoción total de la obra, la armonía integral de la concepción, el alma misma de la obra que analiza" (1923, p. 24). Es evidente que Brenes aspira a un método que trascienda la genética y entre en el terreno de la hermenéutica textual, pero las tecnologías teórico-metodológicas de su época apenas están trabajando para dotar al estudioso de estas herramientas. Quizá por eso se conforma con el atisbo estilístico de su indagación sobre la prosa española.

En el caso del texto de Arturo Agüero Chaves, aunque sin la rigurosidad teórica, al menos, manifiesta el deseo de adscribir su trabajo en la línea de la estilística. De entrada, se disculpa por su poca experiencia como crítico literario y, por ello, aclara que no conoce con detalle los métodos estilísticos más allá de lo rudimentario. Aspira a seguir el camino

Sin embargo, en el contexto de esa primera mitad del siglo $\mathrm{XX}$, es posible señalar algunos atisbos que rompieron con los modelos impresionistas y las formulaciones tradicionales de la preceptiva literaria. Un primer intento aparece en el libro Categorías Literarias de Roberto Brenes Mesén, publicado por Joaquín García Monge en 1923. Brenes discrepa de los procedimientos de análisis que abordan la gramática o las formulaciones retóricas y desacredita, incluso, los principios del estructuralismo, pues propone que frente al fenómeno literario no es posible separar el fondo de la forma. Sin la forma, "ni en el mundo del arte ni en el mundo de la conciencia del artista, inmediatamente anterior a la expresión artística, tiene realidad alguna el fondo. El arte es la forma. El artista es un revelador de la divina belleza de la forma; es la forma misma. Todo cambia cuando cambia la forma" (Brenes Mesén, 1923, p. 34). Si bien, sigue siendo una posición idealista, lo particular es que intenta plantear una refutación crítica con argumentos académicos. Otro caso singular es el texto titulado Sobre los estudios estéticos de Rafael Estrada, publicado en 1926, que da cuenta de una conferencia de naturaleza filosófica dictada a un grupo de estudiantes el 26 de mayo de ese año. En dicha alocución Estrada retoma algunas de las ideas de Benedetto Croce y principalmente de Ernst Meumann. Considera que la Historia y la Psicología son disciplinas esenciales para la interpretación del objeto artístico. No obstante, a pesar de la alusión a Croce, desconoce los alcances de la estilística. 
de la estilística pues, "al estudiar la obra del señor Baudrit, he sentido al menos una pinta de responsabilidad para no caer en ciertas rutinas tradicionales" (1956, p. 294). Esta intención evidencia que el profesor Agüero aspira a un método crítico y serio, y aunque no es experto en metodologías interpretativas, entiende que la estilística es la mejor garantía que puede ofrecer. Este gesto significa que, en dicho contexto, esta tecnología de análisis gozaba de prestigio en la academia, al punto que se consideraba como la mejor opción frente a los mecanismos retóricopreceptivos o las elocuencias del impresionismo.

El texto de Agüero es un estudio amplio en el que intenta explicar distintas particularidades "estilísticas" de la obra de Fabio Baudrit. Entre los tópicos que trabaja están la estructura narrativa, el tratamiento del tiempo, la morfología, la constitución de los diálogos y los motivos dominantes. Según sus palabras: "he trabajado [...] ciertos conceptos elementales del contenido, algo acerca de la construcción, un poco de la técnica narrativa y algunas indicaciones respecto de la estructura" (1956, p. 321). Para la última parte, promete trabajar las formas lingüísticas y, entre ellas, incluye la sonoridad. Es interesante en este punto la relación que establece entre lo sonoro y el rasgo estilístico, pues entiende, como lo hacía tradicionalmente la preceptiva, que el estilo se asocia a la elegancia y la corrección formal. Por esta razón, el escritor, según Agüero, debe evitar la cacofonía y las aliteraciones disonantes, entre otros vicios. Para dar cuenta del estilo de Baudrid, muestra algunas figuras de dicción y, de seguido, algunos de los tropos literarios. Es evidente que el manejo que tiene Agüero de la estilística es limitado. Esto se nota en esta última parte donde no consigue desligarse del todo de algunas de las "rutinas tradicionales" de la retórica. No obstante, los atisbos estilísticos presentes, sobre todo en la primera parte, evidencian el manejo de algunas nociones básicas. En efecto, Agüero incluso propone una tesis tácita: la idea de que la obra narrativa de Baudrit se caracteriza por la concentración y la tensión horizontal. En buena medida, a lo largo del trabajo, esta tesis se comprueba.

Como ya indiqué, el libro Estilística del lenguaje costarricense de Abelardo Bonilla constituye el trabajo de mayor conciencia teórica escrito en Costa Rica en el marco de la estilística. Es pionero no solo de esta disciplina en particular, sino a propósito de las nuevas tecnologías de análisis discursivo que funcionaban en la academia universitaria a mediados del siglo XX. Bonilla tiene claro el papel fundacional de Chales Bally y los aportes esteticistas de los alemanes Vossler y Spitzer; así como las ideas concordantes de Amado y Dámaso Alonso. Cita además a otros autores como Curtius, Hatzfeld, Spoerri, Marouzeau y Bachelard, que le servirán también como fuentes teóricas para su trabajo.

Según Bonilla, la nueva estilística tiene medio siglo de existencia y dispone de más de dos mil obras solo en lo que se refiere a las literaturas románticas. Lamenta que, a pesar de este progreso, esta disciplina no ha tenido mucho éxito en Costa Rica, excepto los trabajos iniciales ya citados. De ahí que su propósito es contribuir al mejor conocimiento y comprensión de este país y su modo de vida mediante la estilística, y de paso, "abrir una brecha en este fecundo campo de investigación, proponiendo con ello una nueva dimensión de los estudios críticos sobre nuestra producción literaria" (1967, p. 5). Se propone el ambicioso plan de un estudio estilístico del lenguaje costarricense "analizando las características propias y distintivas, para deducir de ellas los rasgos esenciales del carácter y modo de ser del hombre de nuestro país" (1967, p. 6). Según su tesis, al identificar la expresión lingüística propia de la nación, en su dimensión histórica, es posible comprender las formas literarias. Se pregunta si esta empresa es posible mediante la estilística y al respecto responde que es factible pues la literatura es el 
“mejor espejo de una nación". La base ideológica de la tarea que busca abarcar el "espíritu de la nación" y los movimientos de la sensibilidad tienen su fundamento en la perspectiva estilística de Charles Bally, quien, como ya he apuntado, sostenía que la estilística abarca el dominio entero del lenguaje. Así el análisis de los fenómenos lingüísticos que incluye sonidos, combinaciones sintácticas y expresiones retóricas, muestran el carácter de la lengua y, por ende, de sus hablantes. Al integrar la totalidad, a través del lenguaje y su estilo, representado en la literatura, el estudioso puede acceder al modo de ser de una nación. Se trata de una pretensión que intenta explicar lo que desde la perspectiva idealista se entendía como el "alma nacional" y que luego, en el marco de las miradas críticas del pensamiento materialista se entendería como "identidad cultural". Para esta tarea, Bonilla integra información histórico cultural y la vincula con la formación literaria y las expresiones lingüísticas coloquiales, "ya que ambas manifestaciones integran plenamente nuestra lengua nacional" (1967, p. 7).

Una novedad en la búsqueda del estilo es la integración del pensamiento psicoanalítico del filósofo francés Gastón Bachelard (1884-1962) y sus estudios sobre la imaginación poética. Los escritos bachelarianos dan cuenta de lo simbólico, principalmente al trasluz de los cuatro arquetipos de la materia, así como las poéticas del tiempo y el espacio. Para Bonilla, la búsqueda de los imaginarios materiales y la interpretación de los mitos ayudan en la identificación de los rasgos estilísticos. Parte de la hipótesis de que, en el caso de Costa Rica, la tierra es el elemento físico más constante, seguido por el agua. En cambio, el aire y el fuego están casi ausentes. Estudia también las significaciones del tiempo y el espacio. Acota que el primero predomina sobre el segundo pues las formas verbales, los modismos y las figuras literarias se inclinan más por las representaciones temporales. Otro de los rasgos que intenta identificar es el tópico afectivo, el que considera pobre a pesar de la denominación de "ticos" (Bonilla, 1967, p. 9).

Bonilla cree que mediante el estudio de la producción literaria también es posible hallar otras características, entre estas, la afirmación de la realidad empírica, la objetividad, el predominio de la inteligencia sobre las vivencias, así como las experiencias sensoriales. Para comprobar sus hipótesis, siguiendo la aspiración totalizante de la estilística, procede de lo externo a lo interno. Según este plan, el primer capítulo lo dedica a la naturaleza, donde ahonda en temas como la tierra y su influencia, el paisaje y el espacio. El segundo, en el que indaga lo interno, estudia el aislamiento individualista de los costarricenses como un efecto colonial. En cada capítulo incluye una selección de ejemplos que toma de obras literarias representativas. En los capítulos siguientes, aborda los motivos literarios coloquiales y el análisis de la expresión literaria, que incluye el estudio de las percepciones sensoriales, sinestesias y cenestesias, metáforas y el sistema verbal, entre otros aspectos. En otro de los apartados, estudia el tema de la afectividad tica y cierra con un estudio sobre el histórico "Pacto de concordia", que dio origen a la Constitución de 1821. A propósito de este último punto, al comparar el discurso político y jurídico con el literario y el coloquial, concluye que:

[...] mientras en nuestra literatura de creación y en el habla corriente predominan lo concreto, lo directo, la tierra y la vida con sus realidades inmediatas, en la literatura oficial -y en todos los escritos que tratan asuntos del Estadopredominan las ideas y las voces de carácter abstracto o indefinido. Esta es la razón del carácter retórico y de la inefectividad de los programas políticos y de los partidos que se apartan del personalismo tradicional, tratando de elevarse a planos ideológicos y doctrinarios de evidente abstracción (1967, p. 76).

A pesar de que Costa Rica es un país joven de menos de siglo y medio, mediante las pruebas discursivas, Bonilla encuentra claves estilísticas que la definen. En su psicología, 
se evidencia la oposición entre la vitalidad telúrica y la actitud estática y conservadora. Se advierten todavía los impulsos juveniles que denotan falta de madurez vital e intelectual. En algunos momentos, el costarricense tiene certeza de su experiencia sensorial y en otros, evidencia temor frente al conocimiento especulativo. "Se busca la voz concreta y el orden lineal y lógico de la frase, pero el estilo impresionista de la mayoría de los escritores se atenúa con notas indeterminadas, como la anteposición de los adjetivos" (1967, p. 78).

Hasta aquí queda claro que Bonilla es el vocero indiscutible de la estilística de la expresión, fundamentada en el análisis de la lengua. Los demás autores costarricenses que utilizan esta herramienta de análisis se inclinan principalmente por la corriente genética. En este proceso, están incluidas una serie de tesis de grado que hicieron los estudiantes universitarios entre las décadas de 1960 a 1980 y otros textos académicos publicados por investigadores universitarios.

De acuerdo con una investigación sobre la crítica literaria aplicada a la narrativa nacional, Duncan, González, Jiménez y Mora (1995) determinaron que la estilística fue una de las metodologías con las que iniciaron profesionalmente los estudios literarios en el ámbito universitario, específicamente en la Universidad de Costa Rica. Entre estos trabajos de graduación que utilizaron la estilística citan los siguientes: Luis Dobles Segreda, memorialista de Heredia (1962) de Humberto Bonilla; Gentes y gentecillas, estudio de una novela (1963) de Jorge Fonseca y La prosa artística de Carlos Salazar Herrera en Cuentos de Angustias y Paisajes (1965) de Jorge A. Camacho (1995, pp. 33-34). Califican que las tesis de este periodo estuvieron marcadas por el análisis de las figuras retóricas y la identificación de autores y obras. Encuentran rasgos del impresionismo, comentarios de texto y opiniones más o menos fundadas sobre las obras. Fijan el año de 1971 como el límite de esta producción. Luego los tesistas se adhieren a otras metodologías, principalmente las estructuralistas. Al revisar de nuevo los títulos y enfoques de estos trabajos de graduación, discrepo del establecimiento de esta fecha, ya que, posterior a 1971, hay otros abordajes aplicados a la narrativa que igualmente utilizan la estilística, tal es el caso de la tesis Análisis estilístico de Mamita Yunai defendida en 1975 por Guillermo Castro Robles. En el género de la lírica también aparecen otras tesis que corresponden a este período. Por ejemplo, Vida y estilo de Porfirio Barba Jacob (1973) de José Antonio Valverde Arguedas y en 1978, Vilma Alfaro Aguilar presenta Algunos recursos poéticos empleados por Jorge Debravo para la expresión de la polaridad: poseedores/ desposeídos en Nosotros los hombres. Un caso más tardío es la tesis Análisis semánticoestructural y estilístico del libro de poemas Vórtices de Jorge Debravo de 1989 elaborada por Roxana González Durán, donde mezcla la estilística con la metodología semántico estructural.

En cuanto a los trabajos publicados por docentes universitarios, en 1989 se divulgan dos trabajos destinados a resumir los planteamientos teóricos de la disciplina. El profesor Jézer González Picado publica un texto titulado Conceptos básicos de la estilística y los profesores Mayra Herra y Francisco Rodríguez editan un material didáctico que titulan La estilística: una opción para el análisis literario. En ambos casos, se trata de iniciativas académicas que recapitulan aspectos teórico-metodológicos. El texto de González enfatiza lo teórico conceptual e incluye, a modo de ejemplificación, el análisis de varios poemas; por su parte, Herra y Rodríguez se centran en el esclarecimiento del modelo clásico sugerido por Spitzer. Este modelo integra una deducción intuitiva que se propone como una tesis inicial y una demostración deductiva que consiste en un estudio detallado del estilo. A partir de estos mecanismos de indagación 
genético-literaria, se publican dos textos adicionales, que aprovechan estos mismos recursos para la explicación de textos. En 1981 Abdenago Cordero Elizondo edita el texto Dos poetas costarricenses al trasluz de la estilística: Lisímaco Chavarría y Jorge Debravo y, a propósito de un homenaje al escritor costarricense, Carlos Luis Fallas, la Asociación de Profesores de Segunda Enseñanza (APSE) publica en el 2002 un texto escrito por Jézer González Picado ${ }^{5}$ en el que analiza la novela Mamita Yunai al trasluz de la estilística, de acuerdo con los preceptos que él mismo había propuesto en su libro de 1989.

\section{Consideraciones finales}

Aunque la estilística, para efectos de los estudios literarios, fue una metodología que buscaba superar las actitudes impresionistas del siglo XIX, su carácter idealista evidenció que no pudo superar el contagio romántico, de ahí que al mismo tiempo bebía de las figuraciones expresivas de la retórica clásica, la preceptiva literaria y las consagraciones al autor como figura clave para encomio del estilo. Como disciplina general de análisis del lenguaje se debatió entre dos corrientes: una (la descriptiva) de mayor interés para los lingüistas y la otra (la genética) más atractiva para los literatos. Aunque nuevos pensadores, como el caso de Michel Riffaterre, Jean Cohen y Pierre Guiraud, entre otros, intentaron darle nuevo aliento, lo cierto es que la estilística no consiguió superarse a sí misma y, a mediados del siglo XX, sucumbió frente a otros modos de enfrentar el lenguaje y el discurso literario.

En el mundo hispano, el magisterio de Damaso Alonso y Amado Alonso hizo que adquiriera un prestigio inusitado, que no consiguieron por la vía de España otras metodologías de la época. De este modo, durante la primera mitad del siglo XX en Hispanoamérica, la estilística suscitó un entusiasmo que se tradujo en un amplio número de publicaciones, ya sea para discurrir sobre sus implicaciones teórico-metodológicas o para aprovechar el mecanismo analítico en la tarea de la explicación de obras literarias. De este modo, autores como Pablo Neruda, por ejemplo, accedieron con mayor facilidad a los altares de la academia.

El prestigio que tenía entonces la estilística en Hispanoamérica obligaba a los profesores universitarios a informarse y formarse en el campo de la nueva disciplina. Esto es evidente también en el ámbito centroamericano, donde destacan las figuras de dos profesoras que dejaron, gracias a sus escritos, una huella significativa en la historia académica. La panameña Elsie Alvarado y la salvadoreña Matilde Elena López promulgaron, aunque desde diferentes puntos de vista, tópicos estilísticos.

En el caso concreto de Costa Rica, el tema del estilo había sido tratado por varios autores en el marco de la preceptiva literaria destinada a la enseñanza secundaria, pero ciertamente la estilística no suscitó el mismo entusiasmo que había generado en otros contextos latinoamericanos. La excepción ocurrirá de manera tardía, pues es en 1967 cuando Abelardo Bonilla publica su libro Estilística del lenguaje costarricense. Aunque hay algunos acercamientos anteriores como los atisbos de Roberto Brenes Mesén y Arturo Agüero Chaves, de manera indiscutible, es este libro de Bonilla el que aborda la estilística bajo un claro marco epistemológico y con la ambiciosa tarea de explicar el estilo del ser nacional mediante los rasgos expresivos de sus lenguajes. Es una tarea que, sin embargo, se desliga de los procedimientos de la escuela española y se adhiere al modelo descriptivo originario de Charles Bally.

$5 \quad$ Este texto se titula Análisis estilístico de Mamita Yunai. 
En 1975, Manuel Picado rinde una especie de homenaje académico a la obra de Amado Alonso. Explica los postulados básicos de las nociones de Alonso bajo la premisa de que su producción merece reconocimiento. Picado es consciente de que la estilística, para ese momento, está siendo sustituida por otras corrientes de análisis discursivo. No obstante, para efectos de Costa Rica, este no sería su obituario. Posterior a esta fecha, otros profesores como Jézer González, Abdenago Cordero, Mayra Herra y Francisco Rodríguez se tomarán el tiempo para explorar de nuevo sus posibles utilidades en el ámbito de los estudios literarios.

Aunque la estilística tocó suelo costarricense de manera tardía, su aliento permaneció casi hasta el final del siglo XX. Su impronta queda en la historia de los estudios filológicos como una de las primeras disciplinas académicas que contribuyó con la tarea de explicar el discurso, especialmente el estilo de nuestra literatura.

\section{Bibliografía}

Agüero Chaves, A. (1956). Estudio epilogal sobre la obra de don Fabio Baudrit G. En F. Baudrit (Ed.), Cifra antológica (pp. 293-341). San José: Trejos hnos.

Alfaro Aguilar, V. (1978). Algunos recursos poéticos empleados por Jorge Debravo para la expresión de la polaridad: poseedores/desposeídos en Nosotros los hombres. (Tesis de grado). Universidad de Costa Rica.

Alvarado de Ricord, E. (1960). Estilo y densidad en la poesía de Ricardo J. Bermúdez. Panamá: Imprenta Nacional.

Alvarado de Ricord, E. (1968). La obra poética de Dámaso Alonso. Madrid: Editorial Gredos. Alvarado de Ricord, E. (1973). Aproximación a la poesía de Ricardo Miró. Panamá: Incude.

Alvarado de Ricord, E. (1978). Rubén Darío y su obra poética. Montevideo: Biblioteca Nacional.

Bally, Ch. (1962). El lenguaje y la vida. (Amado Alonso, Trad.). (4 ed.). Buenos Aires: Editorial Losada. S. A.

Battistessa, A. (1965). El poeta y su poema. Buenos Aires: Editorial Nova.

Bonilla, A. (1967). Estilística del lenguaje costarricense. San José: Publicaciones Universidad de Costa Rica.

Bonilla, H. (1962). Luis Dobles Segreda, memorialista de Heredia. (Tesis de grado). Universidad de Costa Rica.

Brenes Mesén, R. (1923). Las categorías literarias. Cuadernos de pedagogía y otros estudios. San José: Ed. J. García Monge.

Brenes Mesén, R. (1974). El ritmo de la prosa española. En M. E. Dengo (Ed.), Roberto Brenes Mesén (pp. 395-403). San José: Ministerio de Cultura Juventud y Deportes.

Camacho, J. A. (1965) La prosa artística de Carlos Salazar Herrera en Cuentos de Angustias y Paisajes. (Tesis de grado). Universidad de Costa Rica.

Castagnino, R. (1971). El Análisis literario: introducción metodológica a una estilística integral. (3 ed.). Buenos Aires: Editorial Nova. 
Castañeda, F. (1948). Lecciones de Retórica o Literatura Preceptiva. (6 ed.). México: s.e.

Castro Robles, G. (1975). Análisis estilístico de Mamita Yunai. (Tesis de grado). Universidad de Costa Rica.

Cordero Elizondo, A. (1981). Dos poetas costarricenses al trasluz de la estilística: Lisímaco Chavarría y Jorge Debravo. San Ramón: Centro Regional de Occidente, Coordinación de Investigación.

D’Alton, C. (1990). Literatura y Lengua. Ensayos didácticos. San José: EUNED.

Duncan, Q., González, J., Jiménez G. y Mora, M. (1995). Historia crítica de la narrativa costarricense. San José: Editorial Costa Rica.

Estrada, R. (1926). Sobre los estudios estéticos. San José: Imprenta Alsina.

Fernández Retamar, R. (1958). La Idea de la estilística. La Habana: Departamento de Relaciones Culturales.

Fonseca, J. (1963). Gentes y gentecillas, estudio de una novela. (Tesis de grado). Universidad de Costa Rica.

Gómez R., F. (1999). La crítica literaria del siglo XX. Madrid: EDAF.

González Durán, R. (1989). Análisis semántico-estructural y estilístico del libro de poemas Vórtices de Jorge Debravo. (Tesis de grado). Universidad de Costa Rica.

González Picado, J. (1989). Conceptos básicos de estilística. San José: Editorial Fernández Arce.

González Picado, J. (2002). Análisis estilístico de Mamita Yunai. San José: APSE.

Herra, M. y Rodríguez, F. (1989). La estilística: una opción para el análisis literario. San Ramón, Costa Rica: Sede de Occidente.

López, M. E. (1973). Estudios sobre poesía. San Salvador: Ministerio de Educación. Dirección de Publicaciones.

López, M. E. (1974). Interpretación social del arte. San Salvador: Ministerio de Educación. Dirección de publicaciones.

Martín, J. L. (1973). Crítica estilística. Madrid: Editorial Gredos.

Muñoz Meany, E. (1951). Preceptiva literaria. Guatemala: Ministerio de Educación Pública.

Picado, M. (1975). La estilística de Amado Alonso. Revista de Filología y Lingüística, 1(2), 97-102.

Reyes, A. (1944). El deslinde: prolegómenos a la teoría literaria. México: Colegio de México, Centro de estudios literarios.

Reyes, A. (1945). Tres puntos de exegética literaria. Jornadas. (vol. 38). México: Colegio de México, Centro de Estudios Sociales.

Valverde Arguedas, J. A. (1973). Vida y estilo de Porfirio Barba Jacob. (Tesis de grado). Universidad de Costa Rica. 
Villalobos, C. (2010). La obra crítica de Matilde López: los espejos sociales de la literatura. Cultura, (104), 51-59.

Vincenzi P., M. (1939). La enseñanza del estilo. San José: Imprenta Española Soley y Valverde.

Vincenzi P., M. (1940). Perspectiva literaria y composición. San José: Imprenta Lehmann.

Zamora Elizondo, H. (1931). Orientación literaria. San José: Imprenta Alsina. 